\section{European Medical Research Group (Meeting held on 6 June 1989)}

The European Medical Research Group met at the Medical Society of London, Lettsom House, on 6 June 1989. The guest speaker, Professor A.M. McGregor, gave a keynote lecture on "Autoimmunity and the Thyroid".

Following a period of questions and discussions, a poster session was held demonstrating the research in progress of some members of the Group. Their abstracts are published below.

\section{Control of force generation in a human accessory respiratory muscle. (Abstract)}

\section{A.K. Datta}

\section{Department of Medicine, Charing Cross \& Westminster Hospital Medical School, Fulham Palace Road, London W6 $8 R F, U K$.}

Twenty two sternomastoid motor units in 10 normal subjects were studied, using needle electrodes. During eupnea and mild overventilation, the units were inactive. When resistance to inspiration was added, regular discharge of the units occurred mainly during inspiration and sometimes early expiration. Recruitment threshold of the units (\% of inspiratory duration at which the units first discharged regularly), was $0.5-51.2$ (mean 12.0 s.d. 7.8 ); this indicates that the units were involved in flow generation against the inspiratory resistance $\left(7-425 \mathrm{~cm} \mathrm{H}_{2} \mathrm{O}\right.$. litres ${ }^{-1}$.s, mean $53 \mathrm{~cm}$ $\mathrm{H}_{2} \mathrm{O}$. litres ${ }^{-1}$.s often before any appreciable volume had been inspired. This is confirmed by the low mean inspiratory flow rate at recruitment $\left(0.71\right.$ litres $\left.^{-1} . \mathrm{s}\right)$ despite the negative mean mouth pressure $(\mathrm{Pm})$ generated $\left(21.6 \mathrm{~cm} \mathrm{H}_{2} \mathrm{O}\right)$. Following recruitment, most units fired at a steady rate (mean Interspike interval $52.9 \mathrm{~ms}$. s.d. 19.0), despite $\mathrm{Pm}$ becoming more negative, suggesting that recruitment of other units rather than rate coding of units already active is how additional force is generated. In 3/22 units, discharge started with a consistently short $(<10 \mathrm{~ms})$ interval. Such discharge is seen in a variable proportion of human motor units, in different muscles.' This allows optimal force to be generated without the loss of precise control. ${ }^{2}$ Long term airways obstruction may alter the strategies used by the central nervous system in controlling force generation in the accessory muscles.

\section{References}

1. Denslow, J.S. Double discharges in human motor units. $J$ Neurophysiol 1948, 11: 209-215.

2. Stein, R.B. \& Parniggiani, F. Optimal motor patterns for activating mammalian muscle. Brain Res 1979, 175: 372-376.

\section{Estimation of tacrine cholinesterase inhibition. A preliminary report. (Abstract)}

\section{Berti, R. Levy and R.M. Marchbanks}

Department of Old Age Psychiatry and Department of Biochemistry, Institute of Psychiatry, De Crespigny Park, London SES UK.

There is recent evidence from clinical trials that tetrahydroacridine (THA) administered orally can influence positively the cognitive functions in patients suffering from Alzheimer's disease (AD). ${ }^{\prime}$ These effects might be related to inhibition of the activity of cholinesterase (ChE) in the central nervous system and thus increasing the level of acetylcholine in those brain areas related to memory that have been demonstrated to be affected in AD.

There is little knowledge of the pharmacokinetics of THA and the treatment is still based on the correlation between the dosage of the drug and the clinical evaluation of the amelioration of symptoms. The concentration of THA and/or its metabolites in plasma and/or urine has not been correlated to the cognitive effects.

We report the application of a colorimetric technique ${ }^{2}$ to detect the presence of THA and/or its metabolites in readily available biological fluids by evaluating the $\mathrm{ChE}$ inhibition in urine of patients ( 2.1 male aged 74 years and 1 female aged 72 years) undergoing a clinical trial with THA. The urine of patients receiving a single daily dose of $25 \mathrm{mg}$ THA were collected 12 hours following each oral administration of the drug.

With the colorimetric enzymatic assay used the property of the urine to inhibit the activity of purified $\mathrm{ChE}$ (Sigma), was evaluated.

Initial results suggest that almost complete $\mathrm{ChE}$ inhibition is obtained on day 4 of treatment $(87 \pm 3.7 \%$ s.e.m.). The inhibition of $\mathrm{ChE}$ gradually decreased and reached a value of $63 \pm 9.6 \%$ on day 12 . Control urine of patients was collected prior to the beginning of the trial and did not possess any capability to inhibit ChE. Urine tests were also carried out routinely during the trial.

The preliminary results support the use of the colorimetric assay as a useful method for the evaluation of THA and/or its metabolites in readily accessible biological fluids. Furthermore the data obtained so far support previous findings indicating powerful ChE inhibitory properties of THA. Finally the enzymatic method used has provided a simple and reliable test to check patient's compliance during the trial.

\section{References}

1. Summers, W.K., Majovski, L.V., Marsh, G.M., Tachiki, K. \& Kline, A. Oral tetrahydroaminoacridine in long term treatment of senile dementia. $N$ Engl $J$ Med 1986, 315: 1241-1244.

2. Ellman, G.L., Courtney, K.D., Andresjc, V. \& Featherstone, R.M. $A$ new and rapid colorimetric determination of cholinesterase activity. Biochem Pharmacol 1961, 7: 88-95. 


\section{Single European Act: Implications for medicine after 1992. (Abstract)}

\section{D.R.J. Singer}

European Medical Research Group, c/o Fellowship of Postgraduate Medicine, 6 St Andrew's Place, London NWI $4 L B, U K$.

The single European Act' defines new objectives and powers of the European Community (EC). A survey was conducted to assess insight among physicians in training into the implications of the Act for medicine in Europe after 1992. Copies of a questionnaire are being sent to physicians in training on the mailing list for the European Medical Research Group meetings (259) as well as to special interest groups. Thirty replies have been received to date, 25 from UK nationals, 5 from elsewhere. This clearly represents a highly selected sample: however, the preliminary results are of interest. Only 4 of the 30 respondents admitted to any knowledge of the changes which may occur after 1992. Most of these mentioned free movement of doctors within the EC, which has already been in force since 1977 . Twelve of the 25
UK nationals reported no working knowledge of other EC language(s). All 5 foreign nationals reported working knowledge of at least one further non-native language other than English. The majority of respondents would be prepared to spend time learning another EC language, and expressed interest in spending part of their training elsewhere in Europe.

The Act aims to reduce disparities between the various regions and backwardness of the least favoured regions and to extend free movement to all professions within the EC.' However, most EC members do not have an effective manpower planning policy for health care. Pro-EC directives of the European Parliament no longer require unanimous approval of the member countries. The Act is therefore likely to have a major impact on health care. The initial evidence from this study suggests that young physicians are interested in the implications of the Act but appear to be ill-equipped to take advantage of its provisions.

\section{References}

1. Single European Act (1986), Treaty Series No. 31 (1988), Cm 372, HMSO, London. 